\title{
Ethylene response factors regulate expression of HbSUT3, the sucrose influx carrier in laticifers of Hevea brasiliensis
}

\author{
Yi Zhang 1 ${ }^{1}$, Lusheng Xin ${ }^{1}$, Julien Pirrello ${ }^{2,3}$, Yongjun Fang ${ }^{4}$, Jianghua Yang ${ }^{4}$, Jiyan $Q i^{1}$, \\ Pascal Montoro ${ }^{2,3,5}$ and Chaorong Tang ${ }^{1,5}$
}

\begin{abstract}
${ }^{1}$ Natural Rubber Cooperative Innovation Center of Hainan Province \& Ministry of Education of PRC, College of Tropical Crops, Hainan University, 58 Renmin Avenue, Haikou 570228, China; ${ }^{2}$ CIRAD, UMR AGAP, 389 Avenue d'Agropolis - TA A-108/03, F-34398 Montpellier, France; ${ }^{3}$ AGAP, Univ Montpellier, CIRAD, INRA, Montpellier SupAgro, 389 Avenue d'Agropolis - TA A-108/03, F-34398 Montpellier, France; ${ }^{4}$ Rubber Research Institute, Chinese Academy of Tropical Agricultural Sciences, 4 West Xueyuan Road, Haikou 570100, China; ${ }^{5}$ Corresponding authors C.Tang (chaorongtang@126.com or chaorongtang@hainanu.edu.cn); P. Montoro (pascal.montoro@cirad.fr)
\end{abstract}

Received September 16, 2020; accepted December 26, 2020; handling Editor Chung-Jui Tsai

Natural rubber is an important industrial raw material and is commercially produced by rubber trees (Hevea brasiliensis). The sucrose transporter HbSUT3 plays an essential role in rubber production. Its expression in latex (cytoplasm of rubberproducing laticifers) is induced by bark treatment with Ethrel, an ethylene releaser, and the inducing effect correlates well with Ethrel-stimulated rubber yield increase. However, the mechanisms of ethylene induction on HbSUT3 expression are not known. Here, five Ethylene Response Factor (ERF) genes were identified from the cDNA library of Hevea latex by yeast one-hybrid screening with the promoter of HbSUT3 gene as bait. As revealed in a tobacco (Nicotiana tabacum) protoplast transient expression system, these HbERFs were mainly localized in the nucleus and four of them exhibited apparent transactivation activity. Of the five HbERF genes, HbERF-IXc4 was the most frequently screened in yeast onehybrid, accounting for $65 \%$ of the ERF clones obtained. Moreover, among the five HbERFs, HbERF-IXc4 showed the strongest transactivation capacity when expressed in tobacco protoplast, the highest transcript abundance in latex and a close expressional correlation with its target gene, HbSUT3, in response to the Ethrel treatment. Taken together, our results indicate that ERFs, especially HbERF-IXc4, are critically involved in the activation of HbSUT3 expression in latex after Ethrel treatment on Hevea bark, and thus the stimulated latex yield.

Keywords: ethylene signaling, gene expression, rubber biosynthesis, sucrose transporter, transcription factor.

\section{Introduction}

Natural rubber (cis-1, 4-polyisoprene), an important industrial raw material, is commercially harvested from a tropical tree species, Hevea brasiliensis (para rubber tree), by way of tapping, viz. cutting periodically (commonly every 3 days) the bark of tree trunk, and hence severing the rings of rubber-producing laticifers wherein. The rubber production can be stimulated by ethylene, which has resulted in the routine application of Ethrel (2-chloroethyl phosphonic acid), an ethylene releaser, on trunk bark in rubber plantations (Zhu and Zhang 2009). A vast number of physiological and molecular studies have demonstrated that Ethrel application accelerates the supply and metabolism of sucrose in laticifers (Dusotoit-coucaud et al. 2009, Liu et al.
2015) and prolongs the time of latex flow (Tungngoen et al. 2009), and thus increases the latex yield of rubber trees (Zhu and Zhang 2009).

Rubber biosynthesis begins with the sucrose as precursor molecule, and covers $>20$ enzymatic reactions in laticifers (Sando et al. 2008). Due to the symplasmic isolation of laticifers from the neighboring cells/tissues, a sucrose transporter (SUT) has long been hypothesized for sucrose loading into laticifers, and to determine the carbon source for natural rubber biosynthesis and rubber yield (Tupý 1985). Studies on SUT genes in Hevea tree were first conducted independently in two research groups and a total of six distinct SUT genes have been cloned and characterized (Jianghua et al. 2007, 
Dusotoit-coucaud et al. 2009, Dusotoit-coucaud et al. 2010, Tang et al. 2010). Among the six Hevea SUTs, HbSUT3 (=HbSUT1B) is identified as the responsible SUT member in sucrose loading into laticifers in regularly tapped Hevea trees (Tang et al. 2010). Both HbSUT3 expression in latex and latex production are stimulated by ethylene treatment, and more importantly, the latex yield correlates significantly with the stimulating effect of ethylene on HbSUT3 expression (Dusotoitcoucaud et al. 2010, Tang et al. 2010). A recent study reports that HbSUT3 expression correlates positively with the latex yield of Hevea varieties and its expression in seedling bark can serve as an indicator in the early selection for high-yielding clones in Hevea breeding programs (Klaewklad et al. 2017). However, the molecular mechanisms by which ethylene generates inducement on HbSUT3 expression in laticifers are still not known.

Plant SUTs are membrane-localized, energy-dependent proton/sucrose symporters, and contain 12 conserved transmembrane $\alpha$-helices separated by a central cytoplasmic loop (Sauer $2007)$. They play a central role in the regulation of sourceto-sink sucrose transport that determines plant growth and development (Durand et al. 2018). Based on the involvement in different sucrose translocation steps, SUTs can be classified into three types: plasma membrane efflux carriers, plasma membrane influx carriers and tonoplast carriers (Lemoine 2000). HbSUT3 is a high sucrose affinity transporter and belongs to plasma membrane influx carriers (Tang et al. 2010). Its Arabidopsis ortholog, AtSUC2, is highly expressed and involved in phloem sucrose loading in leaves, stems and roots (Truernit and Sauer 1995, Durand et al. 2018).

Expressions of sugar transporter genes are regulated by different transcription factors that vary with plant species and experimental conditions, and are implicated widely in plant growth and development. The companion cell-specific expression of AtSUC2 is determined by conserved cis-regulatory elements for DNA-binding-with-one finger and homeo-domainleucine-zipper transcription factors (Schneidereit et al. 2008). Abscisic acid (ABA)-responsive transcription factor MdAREB2 directly activates the expression of amylase and sugar transporter genes to promote soluble sugar accumulation in apple (Ma et al. 2017). Rice transcription factor OsDOF11 modulates the expression of OsSUT1 and two Sugars Will Eventually be Exported Transporters (SWEET) genes, OsSWEET11 and OsSWEET14 (Wu et al. 2018). HbEIN3/EILs regulate the expression of two sugar transporters, which is predicted by a genomicwide scanning the binding site of these transcription factors, G-box, in the promoters of rubber tree genes (Wang et al. 2019). Cotton fiber elongation requires sucrose transport into expanding fibers, via regulating the expression of SUT gene GhSWEET 12 by the transcription factor GhMYB 212 (Sun et al. 2019). Pollen tube growth is modulated via a MYB transcription factor MdMYB39L, which functions by regulating the expression of a sugar transporter MdSTP13a in apple (Li et al. 2020b).
A WRKY transcription factor PUWRKY31 induces PUSWEET15 transcription, which affects the accumulation of sucrose in pear fruit (Li et al. 2020a).

Here, five genes encoding ERF transcription factors were identified from $H$. brasiliensis latex by yeast one-hybrid system with the promoter of HbSUT3 as bait. We show that these ERFs, especially one of them, HbERF-IXc4, play a critical role in ethylene induction on HbSUT3 expression, and thus the ethylene-stimulated latex production in Hevea trees. To our knowledge, this is the first report of direct involvement of ERFs in regulating sugar transporter genes in response to ethylene treatment.

\section{Materials and methods}

\section{Plant material}

Rubber tree clone Reyan7-33-97 was cultivated on the plantation of Chinese Academy of Tropical Agricultural Sciences (Danzhou, Hainan province, China). They were 10 years old and regularly tapped for latex in a half spiral pattern every 3 days (S/2, d/3). Different tissues, viz. leaf, seed, latex, bark, root, female and male flowers, were sampled from three individual trees, kept in liquid nitrogen and brought to the lab for immediate RNA isolation or stored at $-80{ }^{\circ} \mathrm{C}$. To study the expression patterns of HbSUT3 and ERF transcription factor genes in latex after the ethylene treatment, 1.5\% (w/w) Ethrel in 1\% (w/w) carboxyl methyl cellulose (CMC) was applied to the trunk bark by spreading just above the tapping panel. The latex was sampled by tapping after 3, 12 and $24 \mathrm{~h}$ of Ethrel treatment, as described by Pujade-Renaud et al. (1994). For each time point, three batches of five trees were used. Another three batches of five trees were treated with $1 \% \mathrm{CMC}$ as a control. Total RNA was extracted from latex samples pooled from five trees, respectively, as described (Tang et al. 2007).

\section{Yeast one-hybrid screening}

To identify the transcription factors that regulate HbSUT3 expression, a fragment of HbSUT3 promoter (HbSUT3p) was used as bait for yeast one-hybrid screening. The HbSUT3 promoter fragment employed was cloned by a modified adaptor-PCR in our previous work (Xin et al. 2012), being 2028 base-pairs long upstream of the initiation codon. HbSUT3p was amplified with the primers of 5'- TCGA GCTCTGGGAGCAACCATTACATCAAAATAGAA-3' (forward) and 5'-AGGTCGACGGGAGTTGGTGGTGAGAAGAGTGTG-3' (reverse), digested with the restriction enzymes of Sacl and Sall and cloned into the Sacl-Sall cleaved bait vector pAbAi (TaKaRa, Beijing, China) by a ligation reaction with T4 DNA ligase. A normalized latex cDNA library was produced from $0.1 \mu \mathrm{g}$ latex mRNA as previously reported (Zhang et al. 2014). The mRNA sample used was isolated from an equal ratio mixture of total RNA from latex samples collected at four different time points 
$(0,3,12$ and $24 \mathrm{~h})$ after Ethrel treatment. Yeast one-hybrid library was screened following the user manuals of Yeast One-Hybrid System-Matchmaker Gold kit (TaKaRa).

\section{Identification of ERF transcription factors in silico}

To annotate the genes screened by yeast one hybrid, their sequences were analyzed firstly by BLAST in the NCBI database (https://www.ncbi.nlm.nih.gov/) and Hevea genome database (http://hevea.catas.cn/home/index) (Tang et al. 2016). Then, conserved domains of HbERFs annotated by BLAST were identified by a Pfam search in the EMBL-EBI database (http://pfam.xfam.org/search). Annotated HbERFs were validated lastly by phylogenetic analysis with characterized Arabidopsis AP2/ERF proteins. Phylogenetic tree was constructed by the Neighbor-Joining method in Mega- $X$, of which the bootstrap method was tested using 1000 replicates. The AtERF amino acid sequences were downloaded from UniProt database (https://www.uniprot.org/). To study the function of these HbERFs, their sequences were analyzed by BLAST and matched to the Hevea AP2/ERF superfamily reported (Duan et al. 2013).

\section{Transcript abundance analysis by real-time PCR}

Several rules were applied for ensuring the accuracy of the analysis. The integrity of total RNA samples after DNase I digested was checked by electrophoresis. The cDNA for detecting gene expression was synthesized from $1 \mu \mathrm{g}$ latex total RNA with RevertAid H Minus First Strand cDNA Synthesis Kit (Thermo Fisher Scientific, Shanghai, China). The primers of HbERFs for real-time PCR were designed close to $3^{\prime}$ side of gene coding sequences (Table S1 available as Supplementary data at Tree Physiology Online), and the primers of HbSUT3 and HbUBC4 for real-time PCR was as previously reported by Tang et al. (2010) and Li et al. (2011), respectively. Each sample was tested with three repeats at the same time for real-time PCR.

Real-time PCR was performed using SYBR Premix Ex Taq ${ }^{\text {TM }}$ kit $^{-}$ (TaKaRa) on a LightCycler 2.0 system (Roche, Shanghai, China). The PCR procedure was performed as follows: pre-denaturation at $94{ }^{\circ} \mathrm{C}$ for $30 \mathrm{~s} ; 45$ amplification cycles of denaturation at $94^{\circ} \mathrm{C}$ for $5 \mathrm{~s}$, annealing at $60^{\circ} \mathrm{C}$ for $20 \mathrm{~s}$ and extension at 72 ${ }^{\circ} \mathrm{C}$ for $20 \mathrm{~s}$; melting curves plotted from $72{ }^{\circ} \mathrm{C}$ to $94^{\circ} \mathrm{C}$ with a heating rate of $0.2^{\circ} \mathrm{C} \mathrm{s}^{-1}$.

The amplifying specificity of primers was double-checked by Sanger sequencing of the PCR product. A new pair of primers was designed for replacement if the PCR product was found to be mingled. The amplification efficiency of each primer pair was evaluated via the same PCR procedure but with gradient diluted latex cDNA as template. The relative abundance of transcripts was calculated according to standard curve method (Morrison et al. 1998). Hevea ubiquitin-protein ligase gene HbUBC4 was used as a suitable internal reference as reported (Li et al. 2011).

\section{Construction of plant expression vectors}

Vector construction was carried out by a Gateway cloning strategy (Suzuki et al. 2005) following the user manuals of Gateway ${ }^{\circledR}$ BP Clonase ${ }^{\circledR}$ ॥ Enzyme mix and Gateway ${ }^{\circledR}$ LR Clonase ${ }^{\circledR}$ Enzyme mix (Invitrogen, Thermo Fisher Scientific). The open reading frames of each $H b E R F$ gene with and without stop codon were amplified with specific pairs of primers containing attB adaptors. The PCR products were inserted into a donor vector pDonr207 by a BP recombination reaction and then each $H b E R F$ was transferred into a destination vector by LR recombination reactions (pDonr207 was a gift from Yihua Zhou, Institute of Genetics and Developmental Biology, Chinese Academy of Science, Beijing, China). Expressions of these HbERFs were driven by a CaMV $35 \mathrm{~S}$ promoter in destination vectors, pEaleygate 104, pMDC83 and pMDC32, which were ordered from the Arabidopsis Biological Resource Center (ABRC, Ohio State University, Ohio, USA). All the sequences of these HbERF-amplifying primers were listed in Table S2 available as Supplementary data at Tree Physiology Online. For subcellular localization analysis of these HbERFs, green and yellow fluorescent proteins (GFP and YFP) were fused at their Cterminus in pMDC83 and N-terminus in pEaleygate 104 vectors, respectively.

Synthetic fragments containing four repeats of GCC-box (GCCGCC) and mutant GCC-box (TCCTCC), as an autoactivation control, were inserted into pMDC107 (ABRC), respectively, for the detection of each HbERF's activity. These vectors that have a GFP reporter driven by the GCC box or mutant GCC box, named, respectively, as pMDC107-GCC or pMDC107-mGCC, were constructed previously (Piyatrakul et al. 2014). The vectors of pMDC107-GCC or pMDC107-mGCC and HbERF expression vectors were co-transformed into tobacco protoplasts for transactivation activity detection.

\section{Subcellular localization and transactivation detection in tobacco BY-2 cells}

Tobacco suspension cell line (Nicotiana tabacum L. cv Bright Yellow2, BY-2) was preserved and cultured by the research group of Cellular \& Molecular Biology of Stress Responses in Tropical Woody Species (BURST) in French Agricultural Research Centre for International Development (CIRAD). The procedures for BY2 culturing, protoplast preparation and transfection were as described (Nagata et al. 1992).

To determine the subcellular localization of HbERFs, the protoplasts were transfected with recombinant $p$ EarleyGate 104 and PMDC83 vectors harboring the HbERFs fused with YFP and GFP, respectively. The transfected protoplasts were observed under fluorescence microscopy (Leica DM6000 B, Germany) and photographed by Hamamatsu camera (Japan). To detect the transactivation activity of each $\mathrm{HbERFs}$, the protoplasts were cotransfected with the PMDC32 vectors inserted with an HbERF and PMDC107-GCC or pMDC107-mGCC. The GFP signal of 
Table 1. HbSUT3 promoter-binding ERF transcription factors identified from yeast one-hybrid screening

\begin{tabular}{|c|c|c|c|c|c|c|c|}
\hline \multirow[t]{2}{*}{ Gene } & \multirow[t]{2}{*}{$\begin{array}{l}\text { No. of } \\
\text { clones }\end{array}$} & \multirow[t]{2}{*}{$\begin{array}{l}\text { Amino acid } \\
\text { residues }\end{array}$} & \multirow{2}{*}{$\begin{array}{l}\text { Annotation } \\
\text { by NCBI } \\
\text { BLAST }\end{array}$} & \multicolumn{2}{|c|}{ Hevea genome (Tang et al. 2016) BLAST } & \multicolumn{2}{|c|}{$\begin{array}{c}\text { Hevea AP2/ERF database } \\
\text { (Piyatrakul et al. 2014) BLAST }\end{array}$} \\
\hline & & & & Gene ID & Identity & Best hit & Identity \\
\hline $\mathrm{HbERF-IXa2}$ & 3 & 267 & ERF & scaffold0206_1492583 & $100.0 \%$ & $H b E R F-I X a 2$ & $99.5 \%$ \\
\hline$H b E R F-I X a 3$ & 1 & 284 & ERF & scaffold0668_444732 & $100.0 \%$ & HbERF-IXa3 & $99.4 \%$ \\
\hline$H b E R F-I X c 4$ & 17 & 219 & ERF & scaffold1015_293137 & $99.7 \%$ & $H b E R F-I X c 4$ & $98.6 \%$ \\
\hline Other genes & 95 & - & \multicolumn{5}{|c|}{$\begin{array}{l}\text { Known or unknown proteins other than genuine interacting transcription factors (Table S6 available as } \\
\text { Supplementary data at Tree Physiology Online) }\end{array}$} \\
\hline
\end{tabular}

transfected protoplasts was detected on a flow cytometry (FACS Calibur II, BD, USA).

\section{Results}

\section{Cloning of transcription factors interacting with HbSUT3 promoter}

The cDNA library generated from the latex mRNA of rubber tree after Ethrel treatment was screened by yeast onehybrid with a $\sim 2 \mathrm{~kb}$ promoter fragment of HbSUT3 as a bait. Our screening generated 121 yeast candidate clones. After subcloning, sequencing and BLAST searching in NCBI and Hevea genomics database (http://hevea.catas.cn/home/i ndex) (Tang et al. 2016), 26 clones represented five distinct Hevea ERF transcription factors, named, respectively, as $\mathrm{HbERF-}$ IXa2, HbERF-IXa3, HbERF-IXc4, HbERF-IXc7 and HbERF-IXc8 as guided by subsequent blasting and phylogenetic analysis, while the remaining 95 were annotated as known or unknown proteins other than genuine interacting transcription factors (Table 1, Table S6 available as Supplementary data at Tree Physiology Online). Among the five HbERFs, HbERF-IXc4 was most frequently identified, with 17 clones in the library screening, which may represent the most abundant transcription factor binding to the HbSUT3 promoter (Table 1). Rapid amplification of cDNA ends (RACE) technology was then used to obtain the full coding sequences of these $H b E R F s$, which were predicted to encode proteins of 147 to 267 amino acid residues (Table S3 available as Supplementary data at Tree Physiology Online). The interactions between the five HbERFs with full open reading frames and the promoter of HbSUT3 were retested as positive by transforming the HbERF preys into the HbSUT3 promoter bait strain (Figure S1 available as Supplementary data at Tree Physiology Online). All five HbERFs belonged to the ERF family of Apetala 2/Ethylene Responsive Factor (AP2/ERF) superfamily, containing only one AP2 domain as revealed by searching HbERF amino acid sequences in EMBL-EBI database (https:// www.ebi.ac.uk/Tools/hmmer/).

A phylogenetic tree constructed with the five HbERFs and the entire Arabidopsis thaliana AP2/ERF superfamily revealed that the five HbERFs were clustered in a single clade of ERF family with eight AtERFs (Figure 1). A BLAST search against a comprehensive Hevea AP2/ERF superfamily database of 114 genes (Piyatrakul et al. 2014) revealed that three of the five $H b E R F$ genes had $>98 \%$ nucleotide identities, respectively, with $H b E R F-I X c 4, H b E R F-I X a 2$ and $H b E R F-I X a 3$ in the database, whereas the two remaining had homologs of $<80 \%$ sequence identity. HbERF-IXc4 shows higher expression in bark compared with other Hevea tissues (Duan et al. 2013, Piyatrakul et al. 2014).

ERF transcription factors transcriptionally regulate the expression of their target genes through AP2 domain binding to a cis-regulatory element, GCC-box, on the promoters (Allen et al. 1998, Franco-Zorrilla et al. 2014). We checked the sequence of HbSUT3 promoter and found a GCC-box in -30 bp position upstream of the initiation codon and downstream of transcription start site (Table S5, Figure S2 available as Supplementary data at Tree Physiology Online), justifying the obtaining of five HbERFs in our yeast one-hybrid screening with the HbSUT3 promoter as a bait (Table 1).

\section{Subcellular localization and transactivation of HbERFs}

The protoplast transient expression system of tobacco BY-2 suspension cells was used to determine the subcellular localization and transactivation activity of the five HbERF transcription factors. For subcellular localization assay, the HbERFs were fused with the GFPs at their C-terminal in the pMDC83 vector, and with the YFPs fused at the N-terminal in the pEarleyGate 104 vector. As shown in Figure 2, HbERF-IXc4, HbERF-IXc7 and $\mathrm{HbERF-IXc8}$ were localized mainly in the nucleus and partially in cytoplasm, while HbERF-IXa2 and HbERF-IXa3 were exclusively localized in the nucleus (Figure 2).

To detect the transactivation activity of the five HbERFs, initiator and reporter vectors were constructed and cotransfected into tobacco protoplasts. In the initiator vectors of pMDC32-HbERFs, expressions of the HbERFs were driven by a double $35 \mathrm{~S}$ promoter (Figure 3A). In the reporter vector of pMDC107-GCC, expression of a GFP reporter was driven by four GCC box repeats, whereas the vector of pMDC107-mGCC was used as a self-activation control 


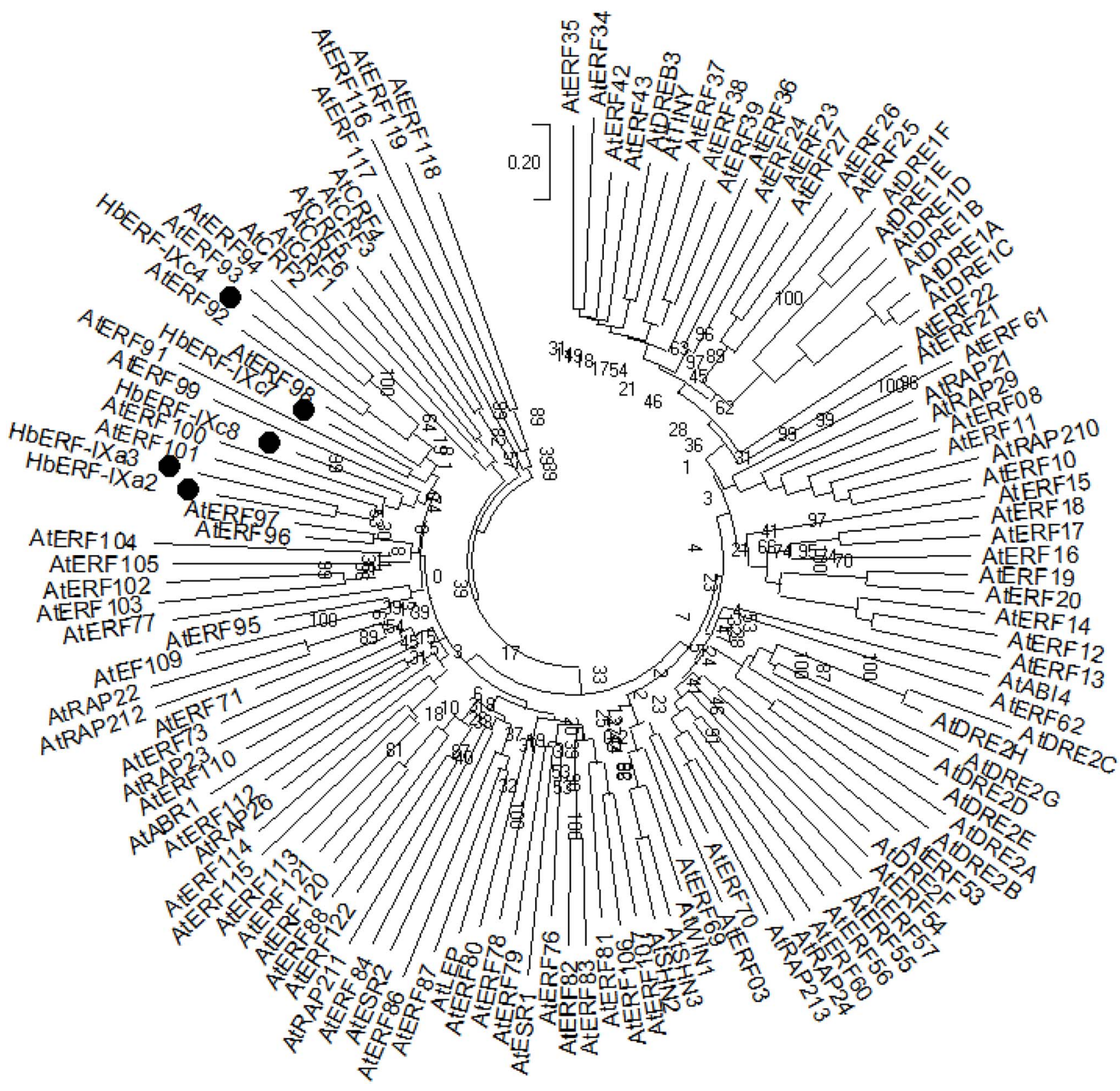

Figure 1. Phylogenetic tree of five Hevea ERFs and the entire Arabidopsis AP2/ERF superfamily. Five Hevea ERFs (HbERFs) are highlighted with a round black dot placed after each member in the tree.

substituted with four mutant GCC box repeats (Figure 3A). Two groups of protoplasts were transfected by pMDC32-HbERFs plus pMDC107-GCC and pMDC32-HbERFs plus PMDC107mGCC, respectively. Taking the blank vector pMDC32 as the background control in the same experiment, the average absolute value of green fluorescence intensity generated from these two groups was detected by flow cytometry as 'pMDC32$X X+G C C$ ' and 'pMDC32-XX + mGCC' ('XX' can be any of the five HbERFs or empty), respectively. Therefore, the relative transactivation activity of a certain $\mathrm{HbERF}$ can be calculated by the following formula: (pMDC32-HbERF + GCC)/ (pMDC32$\mathrm{HbERF}+\mathrm{mGCC})-($ PMDC32 $+\mathrm{GCC}) /(\mathrm{pMDC} 32+\mathrm{mGCC})$
(Piyatrakul et al. 2014). Of the five HbERFs, four (HbERF-IXa2, HbERF-IXa3, HbERF-IXc4 and HbERF-IXc8) showed obvious transactivation activity, with $\mathrm{HbERF}-\mathrm{IXc} 4$ being the strongest (Figure 3B, Table S7 available as Supplementary data at Tree Physiology Online). Interestingly, the remainder, HbERF-IXc7, seemed to be a repressor of reporter activity.

\section{Expressions of HbSUT3 and HbERFs in rubber tree}

To study the tissue-specific expression patterns of HbSUT3 and the five HbERFs, transcripts were investigated by real time PCR (qPCR) in seven Hevea tissues, viz. seed, female flower, male 
HbERF-IXc4

HbERF-IXc7

HbERF-IXa2

HbERF-IXc8

HbERF-IXa3

Gateway cassette

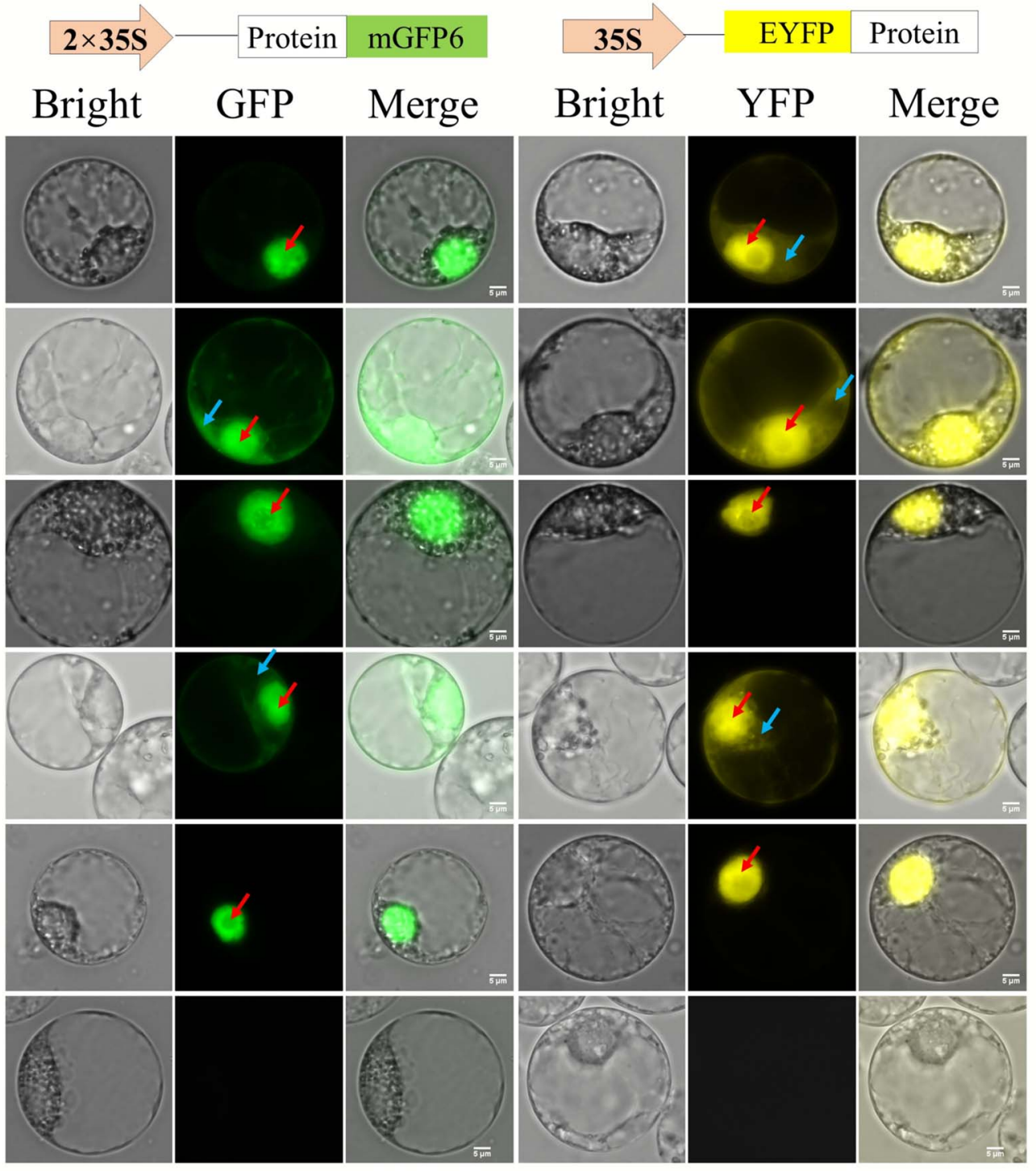

Figure 2. Subcellular localization of HbERFs in tobacco protoplasts. The five HbERF proteins were fused to GFP and YFP in the C-terminus and $\mathrm{N}$-terminus, respectively. The chimeric genes driven by 35S promoter were transiently expressed in BY-2 tobacco protoplast. Subcellular localization was then analyzed by fluorescence microscopy. The bright fields (bright), the corresponding fluorescence channels (GFP or YFP) and the merged pictures are shown. Control cells expressing gateway cassette alone are shown in the bottom panel. The red arrows indicate localization in the nucleus and blue arrows indicate localization in cytoplasm. Bars $=5 \mu \mathrm{m}$.

flower, leaf, trunk bark and latex. As shown in Figure 4, HbERFIXc7 and HbERF-IXc8 were expressed at very low levels in all the tissues examined. HbERF-IXa3 expressed at high levels in the most of the tissues explored, but at very low level in latex. $\mathrm{HbERF-IXc4}$ and $\mathrm{HbERF-IXa2}$ were expressed at moderate levels in most of the tissues examined. Among the five HbERFs, $\mathrm{HbERF-IXc4}$ was the predominant isoform expressed in latex, the expression of which was significantly higher than that of the other four HbERFs together (Figure 4). HbSUT3, the putative target of the five HbERFs, expressed at high levels in female flower and root, moderate in seed, male flower, leaf and latex, but very low levels in trunk bark. It is worth noting that latex (cytoplasm of laticifers) is harvested from trunk bark where the laticifers reside. In this regard, the expression of HbSUT3 has been found to be significantly enriched in laticifers of trunk bark.

To further probe the regulatory roles of HbERF transcription factors in HbSUT3 expression, the expressions of HbSUT3 and five $H b E R F s$ in latex after Ethrel bark treatment were examined by qPCR. As shown in Figure 5, expressions of HbSUT3 and the five HbERFs were all upregulated significantly by the Ethrel treatment. The expression of HbSUT3 in response to Ethrel treatment was consistent with its crucial role in ethylene-stimulated latex production in rubber tree (Dusotoit-coucaud et al. 2010, Tang et al. 2010). Among the five HbERFs, HbERF-IXc4 not only represented the most abundant ERF isoform in latex after the Ethrel treatment, but also exhibited a time course pattern 
A

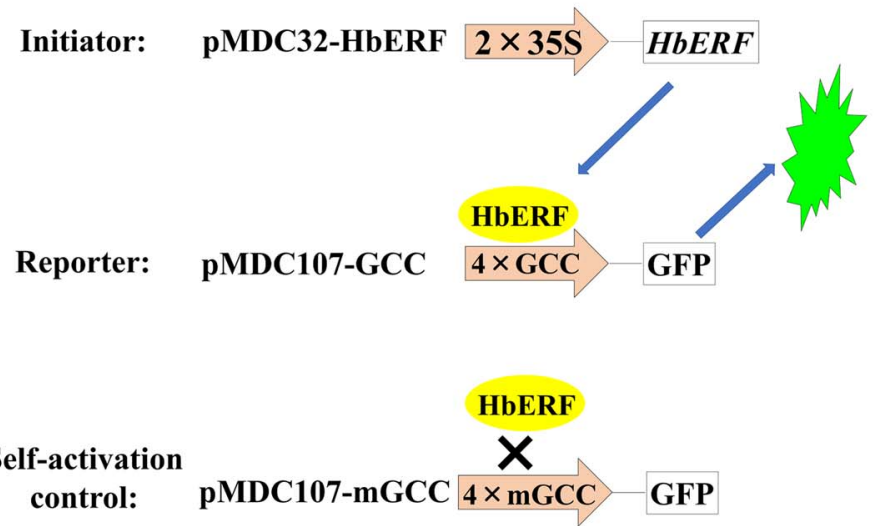

B

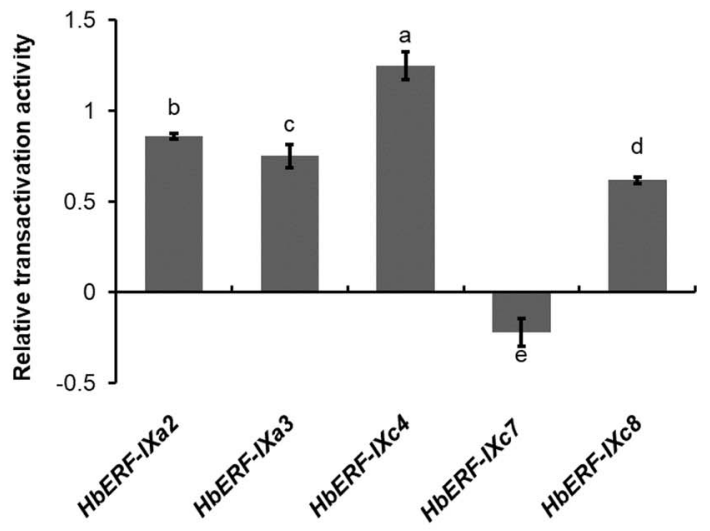

Figure 3. Transactivation activity detection of five HbERFs. (A). Expression cassette in the constructs for transactivation activity detection. (B). Relative transactivation activity of HbERFs. Error bars represent the standard deviation of three independent experiment repeats.

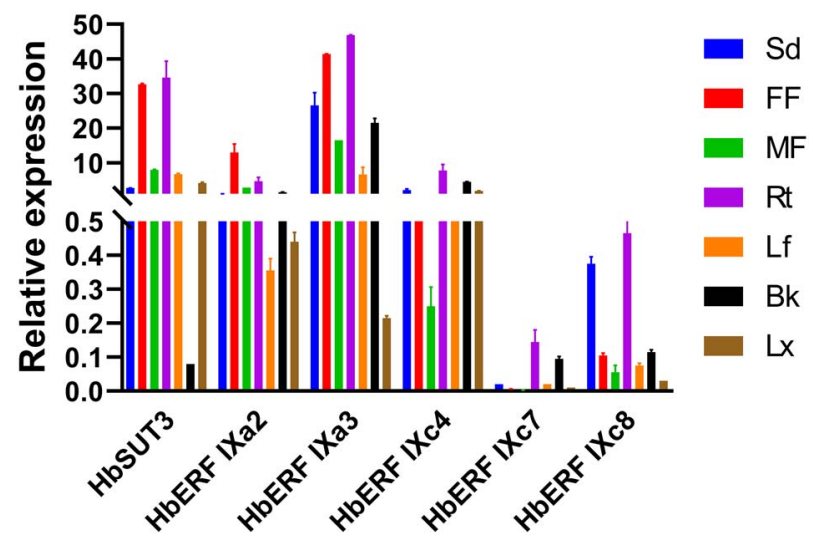

Figure 4. Expressions of HbSUT3 and HbERFs in seven tissues of rubber tree. Transcript levels of HbSUT3 and HbERFs were measured by realtime PCR and normalized by a reference gene $H b U B C 4$. Results are given as the mean of three independent experiments. Error bars represent the standard deviation of three independent experiment repeats. Sd, seed; FF, female flower; MF, male flower; Rt, root; Lf, leaf; Bk, trunk bark; Lx, latex.

of expression similar to HbSUT3 (Figure 5). Similar results of the five HbERF expression patterns were observed in Solexa transcriptome data (Table S4 available as Supplementary data at Tree Physiology Online) (Xiao et al. 2014).

\section{Discussion}

The five HbERFs belong to plant ERF subfamily

The AP2/ERF superfamily members have emerged as key transcriptional regulators of biological processes related to plant growth and development, as well as various stress responses for survival, in which they also respond to multiple hormones, including gibberellins, cytokinins, brassinosteroids, methyl jasmonate, abscisic acid and ethylene (Xie et al. 2019). AP2/ERFs are characterized by containing at least one AP2/ERF DNA binding domain with 40 - 70 conserved amino acids (Sakuma et al. 2002). AP2/ERF superfamily proteins can be classified into three major families defined according to the type of conserved domains contained, with AP2 family proteins containing two repeated AP2/ERF domains, related to ABA Insensitive 3/Viviparous 1 (RAV) family proteins containing a AP2/ERF domain and a B3 DNA binding domain, and ERF family containing a single AP2/ERF domain, respectively (Nakano et al. 2006). The ERF family can be further divided into 12 groups (groups I to X, VI-L and Xb-L), of which groups I to IV and V to $X$ correspond to the Dehydration Responsive Element Binding protein/C-repeat Binding Factor (DREB/CBF) subfamily and ERF subfamily, respectively (Nakano et al. 2006).

The five HbERFs identified by the yeast one-hybrid screening contained a single AP2/ERF domain, therefore belonging to the ERF family under the AP2/ERF superfamily. Phylogenetic analysis revealed the clustering of the five HbERFs with eight AtERFs (AtERF91, AtERF92, AtERF93, AtERF94, AtERF98, AtERF99, AtERF100 and AtERF101) (Figure 1), all of which belong to group IX within the ERF subfamily (Nakano et al. 2006). The ERF subfamily proteins bind to a cis-acting DNA regulatory element, the Ethylene-Response Element (ERE), with AGCCGCC core sequence (GCC-box), and involve in conferring resistance to disease and abiotic stresses (Nakano et al. 2006, Franco-Zorrilla et al. 2014). Some Arabidopsis ERFs (not from group IX) can additionally bind to the cis element of DREBs or C-Repeat Element (DRE/CRT) with A/GCCGAC core sequence on stress-responsive genes, and confer resistance to drought, cold and heat abiotic stresses (Xie et al. 2019). However, the DRE/CRT element is not present in the promoter of HbSUT3, where only a GCC-box was identified (Table S5 available as Supplementary data at Tree Physiology Online). A BLAST search against a comprehensive Hevea AP2/ERF superfamily database further reveals the clustering of the five HbERFs are in group IX under the Hevea ERF subfamily (Table 1). Three group IX HbERFs, i.e., HbERF-IXc4, HbERF-IXc5 and HbERF-IXc6, have 


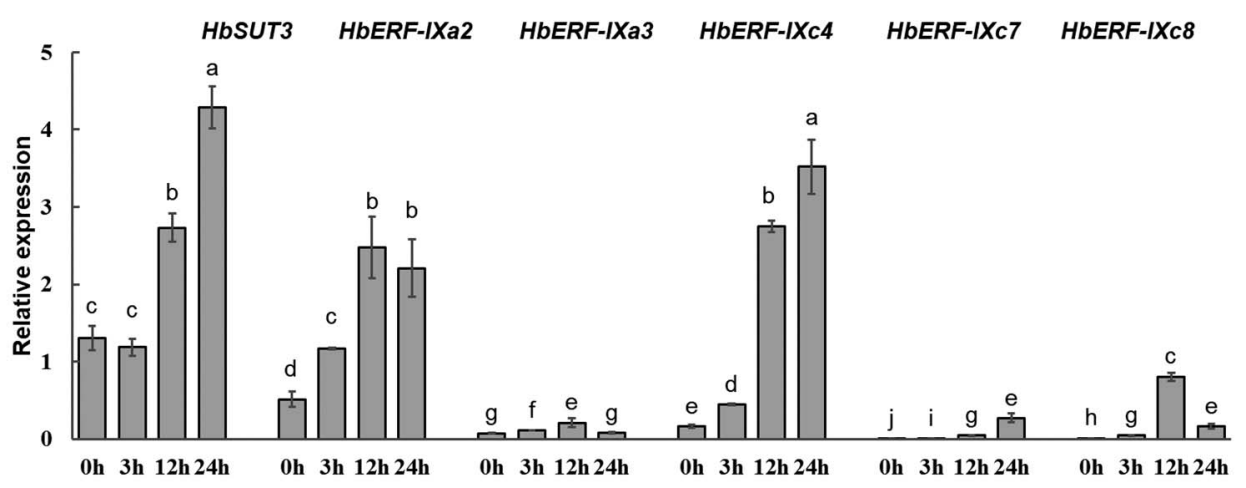

Figure 5. Effect of Ethrel treatment on expressions of HbSUT3 and HbERFs in latex. Transcript levels of HbSUT3 and the five HbERFs were measured by real-time PCR and normalized by a reference gene HbUBC4. Results are given as the mean of three independent experiments. Different labels on the top of columns mean significant difference $(P$-value $<0.05)$. Error bars represent the standard deviation of three independent experiment repeats.

been confirmed containing transactivation activity by binding to the synthetic GCC-box promoters (Putranto et al. 2015). Transactivation activity detection experiments in this study revealed that the five HbERFs bound to GCC-box and activated/repressed the transcription of the GFP reporter gene (Figure 3).

\section{HbERFs are implicated in biological processes related to} rubber production

The ethylene biosynthesis and signaling pathways have been studied in rubber tree for the main reason of understanding ethylene stimulation on latex production (Kuswanhadi et al. 2010). Several biological processes related to rubber production can be stimulated by ethylene treatment in laticifers, such as sucrose loading (Dusotoit-coucaud et al. 2010, Tang et al. 2010), water uptake (Tungngoen et al. 2009) and synthesis of nitrogen assimilation enzymes (Pujade-Renaud et al. 1994). The key regulators in the ethylene signaling pathway, EIN3/EILs, were predicted to target 41 genes involved in rubber biosynthesis and latex flow (Wang et al. 2019). A large number of HbERFs correlate to the special response of rubber trees to ethylene stimulation and harvesting stress (Putranto et al. 2015). A total of 87 ERFs were identified and clustered into 11 groups from rubber tree transcriptome and genomic scaffolds (Duan et al. 2013, Piyatrakul et al. 2014). Transcripts of HbERFs from groups I to VIII are abundant in all the tissues examined and transcripts of HbERFs from group VII are highly accumulated in laticifers (Piyatrakul et al. 2012, Piyatrakul et al. 2014).

HbERF group IX is suggested to be implicated in the crosstalk between ethylene and jasmonate signaling pathways influencing latex production and laticifer differentiation (Duan et al. 2013, Putranto et al. 2015). The transcripts of three genes of group IX, namely HbERF-IXc4, HbERF-IXc5 and HbERF-IXc6, were dramatically accumulated in latex after a treatment combining wounding/jasmonate and ethylene suggesting that these members could be essential integrators of complex hormonal signaling pathways in Hevea (Putranto et al. 2015). The jasmonate signaling pathway is also involved in laticifer differentiation that consists of one of the determining factors affecting rubber production in rubber trees (Deng et al. 2018). Interestingly, the overexpression of $H b E R F-I X c 5$ enhances plant growth and abiotic stress tolerance, and affects laticifer differentiation in transgenic rubber tree lines (Lestari et al. 2018). The five HbERFs identified in this study were defined to ERF group IX (Table 1). Several HbERF-IX members have been previously confirmed as activator-type transcription factors (Putranto et al. 2015). In this study, four of five HbERFs were revealed to be transcription activators, while the remainder, HbERF-IXc7, seems to act as a repressor (Figure 3).

\section{HbERF-IXC4 is actively involved in ethylene-stimulated} HbSUT3 expression in latex

The five HbERFs were identified by yeast one-hybrid screening with the HbSUT3 promoter as bait. They are mainly localized in nucleus and four of them, HbERF-IXa2, HbERF-IXa3, HbERFIXc4 and HbERF-IXc8, are activator-type ERF transcription factors. Four pieces of evidence bolster the roles of HbERF-IXc4 as a critical transcriptional activator of HbSUT3 expression in latex. First, only ERF-type transcription factors are identified in the yeast one-hybrid screening, and more importantly, HbERFIXc4 is the most frequently identified ERF, accounting for $>65 \%$ of the total ERF clones (Table 1). Second, HbERF-IXc4 exhibits much stronger transactivation activity than any other HbERFs examined when assayed in a tobacco protoplast transactivation system (Figure 3). Third, HbERF-IXC4 is the most abundant ERF isoform among these five HbERFs in latex whether or not exposed to Ethrel stimulation (Figure 4, Table S4 available as Supplementary data at Tree Physiology Online). Fourth, dynamic expression of HbERF-IXC4 is most similar to that of HbSUT3 in latex after Ethrel treatment, although expressions of the other 


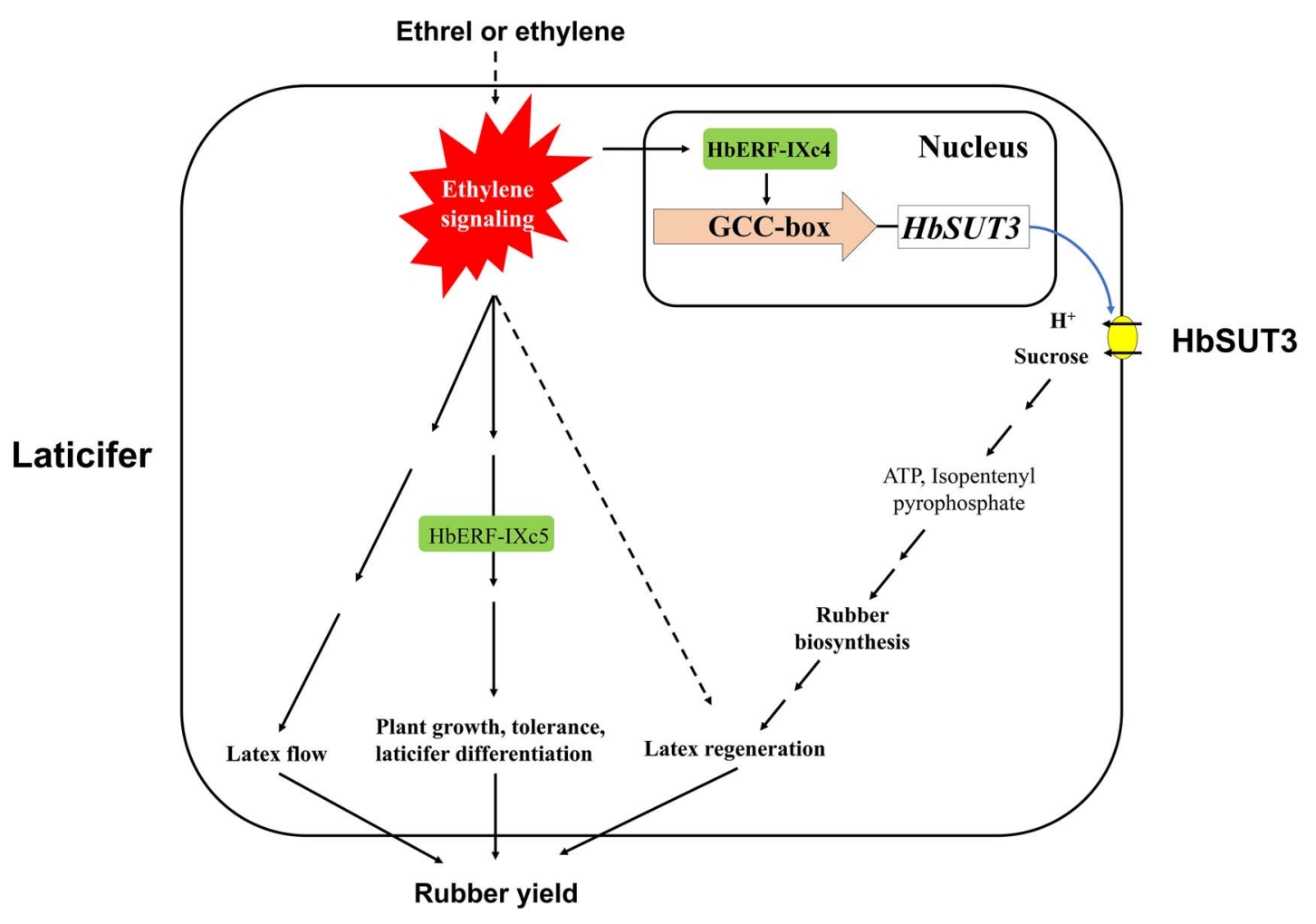

Figure 6. A schematic model of ethylene response factors HbERF-IXc4 (this study) and HbERF-IXc5 (Lestari et al. 2018) in ethylene stimulation of rubber yield in Hevea brasiliensis.

four HbERFs are bolstered as well (Figure 5). Taken together, our results indicate that HbSUT3's expression is regulated by HbERFs, mainly the HbERF-IXc4 in latex after Ethrel treatment. Our work unravels a new way of ethylene regulation on SUT gene expression in higher plants. Considering the key roles of HbSUT3 in the process of sucrose loading in laticifers and ethylene-stimulated rubber production (Tang et al. 2010), we speculate that HbERF-IXc4 plays an important role in enhancing latex production in response to Ethrel stimulation (Figure 6).

\section{Conflict of interest}

The authors declare no competing financial interests.

\section{Authors' contributions}

$Y . Z$. performed most of the experiments, analyzed the data and drafted the manuscript. L.X. constructed the bait vector for yeast one-hybrid screening. J.Y. and J.Q. participated in library construction and field experiments. J.P. and P.M. supervised the experiments of subcellular localization and transactivation activity detection. Y.F. participated in the bioinformatics analysis. C.T. conceived the study and coordinated the project. C.T. and P.M. edited the manuscript, and all authors approved the manuscript.

\section{Supplementary data}

Supplementary data for this article are available at Tree Physiology Online.

\section{Acknowledgments}

This work was supported partially by the National Natural Science Foundation of China (No. 31825007), the Starting Research Fund of Hainan University, and the research unit Genetic Improvement \& Adaptation of mediterraneen and tropical plants of French Agricultural Research Centre for International Development. The authors also thank Julie Leclercq and Florence Dessailly for their kind help on the experiments.

\section{References}

Allen MD, Yamasaki K, Ohme-Takagi M, Tateno M, Suzuki M (1998) A novel mode of DNA recognition by a beta-sheet revealed by the solution structure of the GCC-box binding domain in complex with DNA. EMBO J 17:5484-5496.

Deng X, Guo D, Yang S, Shi M, Chao J, Li H, Peng S, Tian W (2018) Jasmonate signalling in the regulation of rubber biosynthesis in laticifer cells of rubber tree, Hevea brasiliensis. J Exp Bot 69:3559-3571.

Duan C, Argout X, Gébelin V et al. (2013) Identification of the Hevea brasiliensis AP2/ERF superfamily by RNA sequencing. BMC Genomics 14:30-52. 
Durand M, Mainson D, Porcheron B, Maurousset L, Lemoine R, Pourtau N (2018) Carbon source-sink relationship in Arabidopsis thaliana: the role of sucrose transporters. Planta 247:587-611.

Dusotoit-coucaud A, Brunel N, Kongsawadworakul P, Viboonjun U, Lacointe A, Julien J, Chrestin H, Sakr S (2009) Sucrose importation into laticifers of Hevea brasiliensis, in relation to ethylene stimulation of latex production. Ann Bot 104:635-647.

Dusotoit-coucaud A, Kongsawadworakul P, Maurousset L, Viboonjun U, Brunel N, Pujaderenaud V, Chrestin H, Sakr S (2010) Ethylene stimulation of latex yield depends on the expression of a sucrose transporter (HbSUT1B) in rubber tree (Hevea brasiliensis). Tree Physiol 30:1586-1598.

Franco-Zorrilla JM, Lopez-Vidriero I, Carrasco JL, Godoy M, Vera P, Solano R (2014) DNA-binding specificities of plant transcription factors and their potential to define target genes. Proc Natl Acad Sci USA 111:2367-2372.

Jianghua $Y$, Debao $H$, Shujin L, Chaorong T (2007) Six sucrose transporter genes clone and sequences analysis in Hevea brasiliensis. Chin J Trop Crops 28:32-38.

Klaewklad A, Nakkanong K, Nathaworn CD, Nualsri C (2017) Expression of the sucrose transporter 3 (HbSUT3) in rubber tree and its relation to latex yield. Mol Breed 37:1-10.

Kuswanhadi J, Leclercq M, Rio J, Tregear M, Ducamp-Collin N, Montor $P$ (2010) Isolation of three members of the multigene family encoding ACC oxidases in Hevea brasiliensis and investigation of their responses to ethylene stimulation and wounding. J Rubber Res 13:185-205.

Lemoine $R$ (2000) Sucrose transporters in plants: update on function and structure. Biochim Biophys Acta 1465:246-262.

Lestari R, Rio M, Martin F et al. (2018) Overexpression of Hevea brasiliensis ethylene response factor HbERF-IXc5 enhances growth and tolerance to abiotic stress and affects laticifer differentiation. Plant Biotechnol J 16:322-336.

Li H, Qin Y, Xiao X, Tang C (2011) Screening of valid reference genes for real-time RT-PCR data normalization in Hevea brasiliensis and expression validation of a sucrose transporter gene HbSUT3. Plant Sci 181:132-139.

Li XY, Guo W, Li JC et al. (2020a) Histone acetylation at the promoter for the transcription factor PuWRKY31 affects sucrose accumulation in pear fruit. Plant Physiol 182:2035-2046.

Li C, Meng D, Pineros MA, Mao Y, Dandekar AM, Cheng L (2020b) A sugar transporter takes up both hexose and sucrose for sorbitolmodulated in vitro pollen tube growth in apple. Plant Cell 32: 449-469.

Liu S, Lan J, Zhou B et al. (2015) HbNIN2, a cytosolic alkaline/neutralinvertase, is responsible for sucrose catabolism in rubber-producing laticifers of Hevea brasiliensis (Para rubber tree). New Phytol 206:709-725.

Ma QJ, Sun MH, Lu J, Liu YJ, Hu DG, Hao YJ (2017) Transcription factor AREB2 is involved in soluble sugar accumulation by activating sugar transporter and amylase genes. Plant Physiol 174: 2348-2362.

Morrison T, Weis JJ, Wittwer CT (1998) Quantification of low-copy transcripts by continuous SYBR green I monitoring during amplification. Biotechniques 24:954-962.

Nagata T, Nemoto Y, Hasezawa S (1992) Tobacco BY-2 cell line as the "Hela" cell in the cell biology of higher plants. International review of cytology-a survey of cell. Biology 132:1-30.

Nakano T, Suzuki K, Fujimura T, Shinshi H (2006) Genome-wide analysis of the ERF gene family in Arabidopsis and rice. Plant Physiol 140:411-432.

Piyatrakul P, Putranto RA, Martin F, Rio M, Dessailly F, Leclercq J, Dufayard JF, Lardet L, Montoro P (2012) Some ethylene biosynthesis and AP2/ERF genes reveal a specific pattern of expression during somatic embryogenesis in Hevea brasiliensis. BMC Plant Biol 12:244.

Piyatrakul P, Yang M, Putranto RA et al. (2014) Sequence and expression analyses of ethylene response factors highly expressed in latex cells from Hevea brasiliensis. PLoS One 9:e99367.

Pujade-Renaud V, Clement A, Perrot-Rechenmann C, Prevot JC, Chrestin $\mathrm{H}$, Jacob JL, Guern J (1994) Ethylene-induced increase in glutamine synthetase activity and mRNA levels in Hevea brasiliensis latex cells. Plant Physiol 105:127-132.

Putranto RA, Duan C, Kuswanhadi et al. (2015) Ethylene response factors are controlled by multiple harvesting stresses in Hevea brasiliensis. PLoS One 10:e0123618.

Sakuma Y, Liu Q, Dubouzet JG, Abe H, Shinozaki K, YamaguchiShinozaki K (2002) DNA-binding specificity of the ERF/AP2 domain of Arabidopsis DREBs, transcription factors involved in dehydrationand cold-inducible gene expression. Biochem Biophys Res Commun 290:998-1009.

Sando T, Takaoka C, Mukai Y, Yamashita A, Hattori M, Ogasawara N, Fukusaki E, Kobayashi A (2008) Cloning and characterization of mevalonate pathway genes in a natural rubber producing plant, Hevea brasiliensis. Biosci Biotechnol Biochem 72:2049-2060.

Sauer N (2007) Molecular physiology of higher plant sucrose transporters. FEBS Lett 581:2309-2317.

Schneidereit A, Imlau A, Sauer N (2008) Conserved cis-regulatory elements for DNA-binding-with-one-finger and homeo-domainleucine-zipper transcription factors regulate companion cell-specific expression of the Arabidopsis thaliana SUCROSE TRANSPORTER 2 gene. Planta 228:651-662.

Sun W, Gao Z, Wang J et al. (2019) Cotton fiber elongation requires the transcription factor GhMYB 212 to regulate sucrose transportation into expanding fibers. New Phytol 222:864-881.

Suzuki Y, Kagawa N, Fujino T et al. (2005) A novel high-throughput (HTP) cloning strategy for site-directed designed chimeragenesis and mutation using the gateway cloning system. Nucleic Acids Res 33:e109-e109.

Tang C, Qi J, Li H, Zhang C, Wang Y (2007) A convenient and efficient protocol for isolating high-quality RNA from latex of Hevea brasiliensis (Para rubber tree). J Biochem Biophys Methods 70:749-754.

Tang C, Huang D, Yang J, Liu S, Sakr S, Li H, Zhou Y, Qin Y (2010) The sucrose transporter HbSUT3 plays an active role in sucrose loading to laticifer and rubber productivity in exploited trees of Hevea brasiliensis (Para rubber tree). Plant Cell Environ 33:1708-1720.

Tang C, Yang M, Fang Y et al. (2016) The rubber tree genome reveals new insights into rubber production and species adaptation. Nat Plants 2:16073.

Truernit E, Sauer N (1995) The promoter of the Arabidopsis thaliana SUC2 sucrose-H+ symporter gene directs expression of betaglucuronidase to the phloem: evidence for phloem loading and unloading by SUC2. Planta 196:564-570.

Tungngoen K, Kongsawadworakul P, Viboonjun U, Katsuhara M, Brunel N, Sakr S, Narangajavana J, Chrestin H (2009) Involvement of $\mathrm{HbPIP} 2 ; 1$ and HbTIP1;1 aquaporins in ethylene stimulation of latex yield through regulation of water exchanges between inner liber and latex cells in Hevea brasiliensis. Plant Physiol 151:843-856.

Tupý J (1985) Some aspects of sucrose transport and utilization in latex producing bark of Hevea brasiliensis Muel. Arg. Biol Plant 27:51-64.

Wang Q, Xu G, Zhai J, Yuan H, Huang X (2019) Identification of the targets of HbEIN3/ElLs in genomic wide in Hevea brasiliensis. Biosci Biotechnol Biochem 83:1270-1283.

Wu Y, Lee SK, Yoo Y, Wei J, Kwon SY, Lee SW, Jeon JS, An G (2018) Rice transcription factor OsDOF11 modulates sugar transport by promoting expression of sucrose transporter and SWEET genes. Mol Plant 11:833-845. 
Xiao X, Tang C, Fang Y, Yang M, Zhou B, Qi J, Zhang Y (2014) Structure and expression profile of the sucrose synthase gene family in the rubber tree: indicative of roles in stress response and sucrose utilization in the laticifers. FEBS J 281: 291-305.

Xie Z, Nolan TM, Jiang H, Yin Y (2019) AP2/ERF transcription factor regulatory networks in hormone and abiotic stress responses in Arabidopsis. Front Plant Sci 10:228.
Xin L, Yang J, Tang C (2012) An improved promoter-cloning method based on adaptor-PCR and its application in rubber tree. Bull Bot Res 32:296-303.

Zhang Y, Yang J, Liang Q, Tang C (2014) Construction of a normalized Hevea brasiliensis latex cDNA expression library in yeast. Chin J Trop Crops 35:868-871.

Zhu J, Zhang Z (2009) Ethylene stimulation of latex production in Hevea brasiliensis. Plant Signal Behav 4:1072-1074. 